EPJ Web of Conferences 113,04028 (2016)

DOI: $10.1051 /$ epjconf/201611304028

C) Owned by the authors, published by EDP Sciences, 2016

\title{
Neutrino pion production off deuteron
}

\author{
F. Myhrer ${ }^{1, a}$ and S. Pastore ${ }^{1,2, b}$ \\ ${ }^{1}$ Dept. Physics and Astronomy, Univ. South Carolina, Columbia, SC 29208 \\ ${ }^{2}$ Theoretical Division, Los Alamos National Laboratory, Los Alamos, NM 87545, USA
}

\begin{abstract}
Experimental investigations of neutrino properties, using neutrino beams generated at accelerators facilities, necessitate a detailed and precise knowledge of neutrinonucleus reaction mechanisms. In the energy region of nuclear quasi-elastic scattering, pion-production reactions constitute an important background process. A theoretical understanding of these processes is then required in order to correctly determine the produced neutrino energy spectrum. In the first stage of our research project, we study neutrino induced pion-production off deuterons. The choice of the deuteron minimizes the complications of the nuclear dynamics associated with larger nuclear systems. We evaluate the pion-production reaction near threshold using heavy baryon chiral perturbation theory.
\end{abstract}

\section{Introduction}

Neutrino beams generated in accelerator-based neutrino experiments [1] are not mono-energetic. Their energy spectrum spans from a few hundred $\mathrm{MeV}$ to several $\mathrm{GeV}$ and the neutrino energy spectrum is reconstructed from final state observations of neutrino-nucleus reactions. The reconstruction procedure relies on an accurate theoretical understanding of the involved neutrino-nucleus reactions along with a quantitative evaluation of the associated cross-sections. The relevant reactions occurring in this energy regime are nuclear quasi-elastic scattering, pion-productions and deep inelastic scattering. A detailed and precise knowledge of the neutrino-nucleus reaction mechanisms is required in order to determine the neutrino energy spectrum with sufficient accuracy [2].

Theoretical studies of neutrino scattering off light nuclei $(\mathrm{A} \leq 16)$ in the quasi-elastic regime [36], show that many-body terms in both the nuclear Hamiltonian and electro-weak currents provide sizable, non-negligible contributions to the evaluated cross sections. An important background process to the quasi-elastic reactions is generated by neutrino-nucleus pion-production reactions. In the following, we focus on the neutrino induced pion-production reactions off light nuclei near threshold, where an effective field theory approach is applicable. In order to minimize nuclear dynamics effects, we consider pion-production rections in neutrino-scattering off deuterons and focus on charge current reactions $v_{l}+d \rightarrow l+\pi+N+N$.

\footnotetext{
a e-mail: myhrer@physics.sc.edu

be-mail: saori@lanl.gov
} 


\section{The chiral effective field theory approach}

We use heavy baryon chiral perturbation theory (ChPT) to evaluate the transition amplitudes entering pion-production in neutrino scattering off deuteron. ChPT allows a systematic evaluation of high order corrections to the leading order (LO) amplitudes. In this theory, which is applicable for long wave length probes, the short distance hadronic structure and dynamics are parametrized by low-energy constants (LECs). ChPT provides effective interactions that preserve the symmetries and pattern of symmetry breaking exhibited by the underlying theory of QCD. We use an effective hadronic Lagrangian, $\mathcal{L}$, which is written in terms of pion and nucleon fields. It can be expressed as an expansion in $\left(Q / \Lambda_{\chi}\right)^{n} \ll 1$, where $Q$ represents a typical four-momentum transfer in the process under study, or the pion mass, $m_{\pi}$, and $\Lambda_{\chi} \sim 1 \mathrm{GeV}$ is the chiral symmetry-breaking energy scale. The $\mathcal{L}^{(n)}$ terms of order $\left(Q / \Lambda_{\chi}\right)^{n}$ entering the effective Lagrangian can be arranged according to their relevance as:

$$
\mathcal{L}=\mathcal{L}_{\pi \pi}^{(2)}+\mathcal{L}_{\pi N}^{(1)}+\mathcal{L}_{\pi N}^{(2)}+\mathcal{L}_{\pi N}^{(3)}+\cdots
$$

This calculational scheme has been utilized to study electro-induced pion-production rections (see, e.g., [7-9]). Here, we evaluate the neutrino-induced pion-production transition amplitude including up to next-to-next-to-leading order (NNLO) contributions in the chiral expansion. Leading order (LO) terms - or impulse approximation terms--where pions are produced off single nucleons, contain the LO vertices constructed from the the $\mathcal{L}_{\pi \pi}^{(2)}$ and $\mathcal{L}_{\pi N}^{(1)}$ terms in the chiral Lagrangian, for which we take the expressions given in Ref. [10]. NLO and NNLO corrections to the LO one-body operator are evaluated using higher order terms in the chiral Lagrangian, namely $\mathcal{L}_{\pi N}^{(2)}$ and $\mathcal{L}_{\pi N}^{(3)}$, which are taken from Refs. [10,11]. Physically, this corresponds to accounting for nucleon recoil corrections of order $m_{N}^{-2}$. At NNLO one-body currents include also loop corrections to the LO diagrams. The ultraviolet divergences of these loops are regulated by LECs contained in $\mathcal{L}_{\pi N}^{(3)}$. In addition to renormalizing the nucleon mass etc., these loop-diagrams naturally generate lowest order vertex form factors. For example, they generate the nucleon isovector charge form factor expanded to order $q^{2}$-here $q$ is the momentum transferred to the nucleon--where the finite part of the corresponding LEC is given by the isovector charge r.m.s. radius of the nucleon.

Two-nucleon currents enter at NNLO. Hadronic axial currents contributing to the pion-production reaction are evaluated from tree-level diagrams - some of which are illustrated in the panels of Fig. 1-while tree-level hadronic vector currents are derived from the diagrams illustrated in the panels of Fig. 2. It is to be noted that at this order, i.e., NNLO, all the LECs are determined and the theory has predictive power.

The next step in this project, is to evaluate the calculated transition operators sandwiched between initial deuteron wave functions and nucleon-nucleon final scattering states.

\section{Acknowledgements}

This work is supported in part by NSF Grant No. PHY-1068305.

\section{References}

[1] The LBNE Collaboration, arXiv:1307.7335 (2013).

[2] H. Gallagher, G. Garvey and C.P. Zeller, Annu. Rev. Nuc. Part. Sci., 61, 355 (2011); G. Garvey, talk at Fermi National Laboratory (2011), http://minerva-docdb.fnal.gov/cgibin/ShowDocument?docid=5878 .

[3] A. Lovato, S. Gandolfi, J. Carlson, S. Pieper and R. Schiavilla, Phys. Rev. C 91, 062501 (2015). 


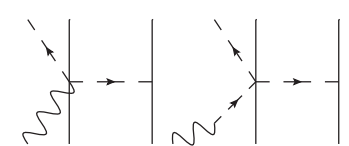

$a 1$

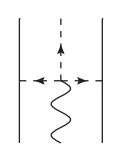

$a 3$

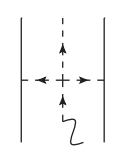

$a 4$

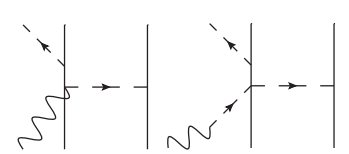

$a 5$

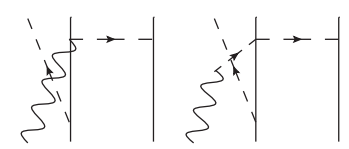

$a 7$

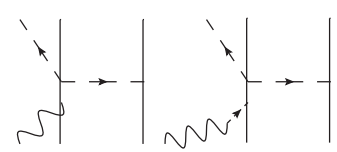

$a 9$

$a 10$

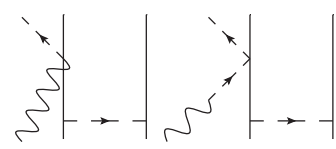

$a 15$

$a 16$

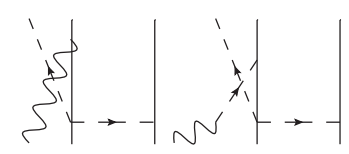

$a 11$

$a 12$

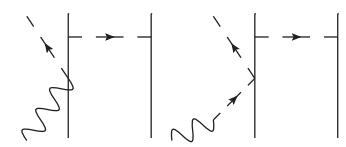

$a 13$

$a 14$

Figure 1. Sample of diagrams contributing to the two-nucleon axial current. The wiggly line represent incoming axial probes, while pions and nucleons are indicated by dashed and solid lines, respectively. The verticies entering the associated amplitudes are derived from $\mathcal{L}_{\pi \pi}^{(2)}, \mathcal{L}_{\pi N}^{(1)}$ and $\mathcal{L}_{\pi N}^{(2)}$ [10].

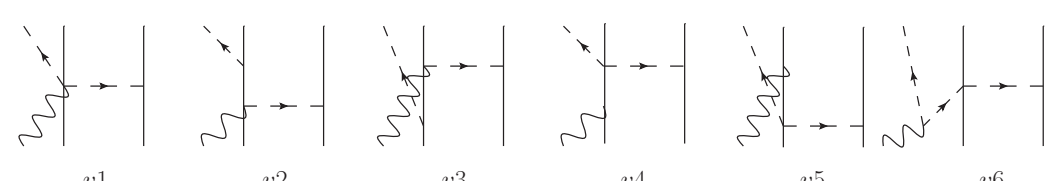

$v 1$

$v 2$

$v 3$

$v 4$

$v 5$

$v 6$

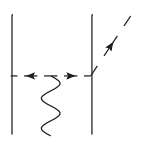

$v 7$

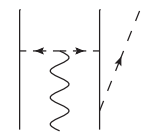

$v 8$

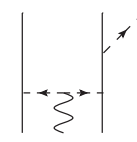

$v 9$

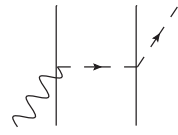

$v 10$

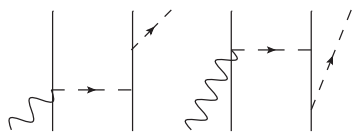

$v 11$

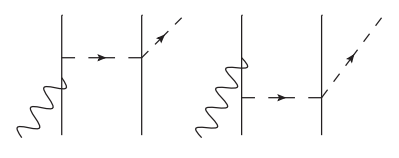

$v 13 \quad v 14$

Figure 2. Diagrams contributing to the two-nucleon vector current. Notation is as in Fig. 1 
[4] M. Martini, M. Ericson, G. Chanfray and J. Marteau, Phys. Rev. C 80, 065501 (2009); M. Martini and M. Ericson, Phys. Rev. C 90, 025501 (2014).

[5] G.D. Megias et al., Phys. Rev. D 91, 073004 (2015).

[6] R. Gran, J. Nieves, F. Sanchez, M.J. Vicente Vacas, Phys. Rev. D 88, 113007 (2013)

[7] S.R. Beane, C.Y. Lee and U. van Kolck, Phys.Rev. C 52, 2914 (1995).

[8] S.R. Beane, V. Bernard, T.-S.H. Lee, Ulf-G. Meißner and U. van Kolck, Nucl.Phys. A 618, 381 (1997).

[9] H. Krebs, V. Bernard, and Ulf-G. Meißner, Nucl.Phys. A 713405 (2003).

[10] V. Bernard, N. Kaiser and U.-G. Meißner, Int. J. Mod. Phys. E 4, 193 (1995).

[11] N. Fettes, U.-G. Meißner and S. Steininger, Nucl. Phys. A 640,199 (1998). 